\title{
Photonic coherent state transfer with Hamiltonian dynamics
}

\author{
S. Weimann, ${ }^{1}$ A. Kay, ${ }^{2}$ R. Keil,${ }^{1}$ S. Nolte, ${ }^{1}$ and A. Szameit ${ }^{1, *}$ \\ ${ }^{1}$ Institut für angewandte Physik, Abbe Center of Photonics, Friedrich-Schiller-Universität Jena, \\ Max-Wien-Platz 1, 07743 Jena, Germany \\ ${ }^{2}$ Department of Mathematics, Royal Holloway University of London, Egham TW20 OEX, UK \\ *Corresponding author: alexander.szameit@uni-jena.de
}

Received November 5, 2013; accepted November 18, 2013;

posted November 25, 2013 (Doc. ID 199090); published December 23, 2013

\begin{abstract}
We report the observation of near-perfect light wave transfer by emulating quantum state transfer on a lattice with Hamiltonian dynamics, i.e., time-dependent intersite couplings. The structure transferring a single waveguide excitation over 11 sites with a fidelity of 0.93 works for classical light as well as single photons. As our implementation of perfect quantum state transfer uses a photonic setting, we introduce polarization as a new degree of freedom to the transport protocol. We demonstrate rotation operations of up to $40^{\circ}$ on polarization during state transfer. (C) 2013 Optical Society of America
\end{abstract}

OCIS codes: (130.3120) Integrated optics devices; (200.3050) Information processing; (130.2755) Glass waveguides; (060.5565) Quantum communications; (270.5585) Quantum information and processing.

http://dx.doi.org/10.1364/OL.39.000123

Feasible quantum computers that are able to outperform classical computation schemes require a large number of qubits [1]. However, maintaining control and coherence over large quantum registers poses serious challenges [2]. A feasible route for overcoming this problem is to divide the large qubit register into smaller units, which interact via so-called "quantum wires." The basic task of the quantum wire is, thus, to coherently transfer a quantum state between the interfaces of two separated subregisters. A possible implementation of this challenging task is the utilization of nearest-neighbor interactions in a discrete lattice, such as chains of spin-1/2 particles $[3,4]$ or waveguide lattices $[5,6]$.

In all state transfer schemes, an initial excitation of a boundary site is transported automatically to the opposite side of the chain due to the intersite coupling [7]. However, in systems with time-independent Hamiltonians (i.e., static coupling), the transfer is accomplished perfectly for one specific moment (transfer time) only. After this moment, the excitation will again spread out over the entire chain. As a consequence, all timeindependent transfer schemes are subject to the limitations that result from precise timing of the extraction of the transferred state in addition to the precise specification of the coupling strengths-the quality of the transfer in these schemes is sensitive to (random and systematic) errors of the fixed intersite coupling $[\underline{5}, \underline{6}, \underline{8}]$.

A solution to this problem is provided by models involving an explicit time dependence of the Hamiltonian, meaning the coupling between the chain sites is changed dynamically. The marginal couplings can be controlled to trap the excitation at the receiver site after perfect state transfer (PST) is accomplished. It has been shown that this can be achieved even if all couplings other than the marginals are constant [9]. There is one particular among these schemes, which turns out to be also robust to imperfections of the couplings [10]. To date, this model is the only time-dependent model that has been formulated analytically for arbitrary lengths $N$ of the chain [10]. Nevertheless, precise temporal control of individual sites is challenging to realize in a spin setting and, hence, experimental demonstration of such a system is still missing.

In our work, we demonstrate the implementation of a time-dependent coherent state transfer scheme using optical waveguides. Our system can be used for the highfidelity transfer of classical as well as quantum light along a chain of waveguides on a chip. Moreover, making use of the degrees of freedom inherent to the optical setting, we elucidate the versatility of the transfer scheme by simultaneously rotating the input polarization during the transfer in a controlled manner. This new functionality is not possible with already investigated time-independent transfer schemes, as the intersite coupling in the optical system is noticeably polarization-dependent. The integrated device described in our work might find application in photonic on-chip quantum computation circuits, as it provides a combination of spatial transfer across chips with a well-defined rotation of the light's polarization.

The model studied in this Letter was originally considered for a chain of coupled spins, which is depicted in Fig. 1(a). The two marginal couplings $C_{A}$ and $C_{B}$ are controlled externally $[9,10]$. This system is closely related to those studied in [11] to provide quantum computation via a Universal Quantum Interface [12]. Each of the $N$ spins in the chain is associated with the qubit

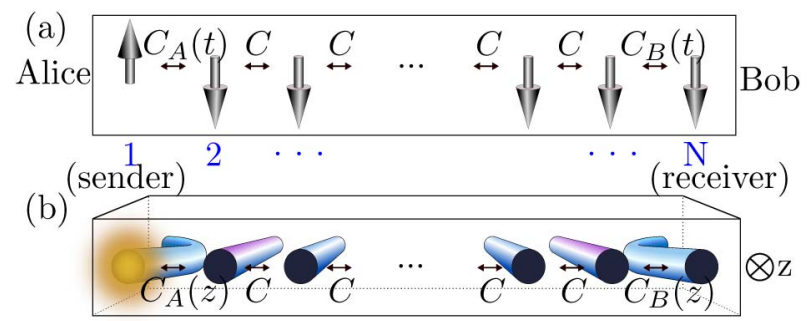

Fig. 1. (a) Coupling distribution of the time-dependent state transfer model. The picture shows the qubit chain just after the initiation of state transfer. (b) The waveguide structure that emulates the excitation evolution in the spin chain. 


$$
\left|\psi_{n}\right\rangle=a_{n}(t)|1\rangle+b_{n}(t)|0\rangle \quad(n=1, \ldots, N),
$$

where $a_{n}(t)$ is the time-dependent probability amplitude for the $n$th spin being "up." In the single-excitation subspace, the transport of amplitudes $a_{n}$ is governed by a Schrödinger equation with a nearest-neighbor interaction Hamiltonian. Except at the edges of the one-dimensional (1D) chain, the model assumes constant and homogeneous couplings of strength $C$. PST is achieved within transfer time $T$ if the chain evolves from $\left[a_{1}, \ldots, a_{N}\right]=$ $[1,0, \ldots, 0]$ at $t=0$ to $\left[a_{1}, \ldots, a_{N}\right]=[0, \ldots, 0,1]$ at time $t=T$.

The implementation of coherent state transfer in arrays of evanescently coupled waveguides is based on the formal analogy between the excitation amplitude $a$ of a spin in a $1 \mathrm{D}$ chain of spin-1/2 particles and the electric field amplitude in a single-mode waveguide of a waveguide array. The fundamental mode of each waveguide can be maximally excited, which corresponds to $|1\rangle$, or dark, corresponding to $|0\rangle$. Consequently, the field amplitude $\Phi_{n}$ in each waveguide can be interpreted as the amplitude $a(t)$ of the associated qubit. As illustrated by the comparison of the optical structure with the quantum chain in Fig. 1, the formal analogy between excitation transfer in spin chains and in waveguide arrays also suggests the mapping of the quantum time evolution onto the spatial propagation of light along the longitudinal direction $z$. The ferromagnetic spin interactions transferring amplitude $a$ from spin 1 to $N$ in the 1D chain are emulated by the evanescent coupling $C$ between waveguides. This coupling decays exponentially as their separation $d$ is increased, i.e.,

$$
C=\kappa \cdot \exp \{-d / \delta\}
$$

where $\kappa$ and $\delta$ are parameters depending on the waveguide properties and the wavelength $\lambda$ of the transfer light [13]. The vector of coupling coefficients can thus be translated into a vector of separations between adjacent waveguides. In order to implement the time dependence of the marginal coupling in the spin chain, the edge waveguides are curved with respect to $z$.

In general, it is a priori not clear whether there exists a set of functions $C_{A}$ and $C_{B}$, which can provide PST. According to a recent work [10], functions $C_{A}(z)$ and $C_{B}(z)$ can be formulated analytically if one demands that the "sender" waveguide release the excitation such that a Gaussian pulse of the form

$$
\Phi_{n}(z)=\frac{1}{\sqrt{\Delta \sqrt{\pi}}} e^{-i \frac{\pi}{2} n} e^{-\frac{1}{2}\left(\frac{n-x_{0}-2 C z}{\Delta}\right)^{2}},
$$

is established in the homogeneous part. This is achieved by using the $z$-dependent couplings to simulate the central region of an infinitely long uniformly coupled chain [9] through which a Gaussian pulse propagates [14]. The Gaussian pulse has width $\Delta$ and is initially centered at a fictitious site $x_{0}$ located in the negative half-space. It is essential that the pulse is propagating with maximal group velocity $v_{g}=2 C$ along the chain, as under this condition, the diffraction of the Gaussian wave packet can be neglected [15], if width $\Delta$ is sufficiently large
[14]. Consequently, the shape of the Gaussian can be assumed to be invariant under $z$-evolution in the homogeneous part, and one finds that for the $z$-dependent coupling at the sender site

$$
C_{A}=\Gamma_{1} \frac{e^{-\frac{1}{2}\left(\frac{2 C z+x_{0}-1}{\Delta}\right)^{2}}}{\left[1-\Gamma_{2}\left(1+\operatorname{erf}\left\{\frac{2 C z+x_{0}-1-0.5}{\Delta}\right\}\right)\right]^{1 / 2}},
$$

where

$$
\begin{gathered}
\Gamma_{1}:=\frac{C}{\sqrt{\Delta} \sqrt{\pi}}, \\
\Gamma_{2}:=\frac{1}{2} e^{-\frac{1}{4 \Delta^{2}}}, \\
\operatorname{erf}(x):=\frac{2}{\sqrt{\pi}} \int_{0}^{x} e^{-t^{2}} \mathrm{~d} t .
\end{gathered}
$$

Assuming the shape invariance of the pulse, the couplings $C_{A}(z)$ and $C_{B}(z)$ are mutually mirror symmetric with respect to $z=T / 2$, resulting in the condition

$$
C_{B}(z)=C_{A}(T-z) \text {. }
$$

Given this symmetry, the transfer distance $T$ can be calculated as

$$
2 C T=N+1-2 x_{0} .
$$

Since $C, T$, and $N$ are given, this formula fixes $x_{0}$, ensuring symmetric evolution. In Fig. 2, an example of $C_{A}(z)$ and $C_{B}(z)$ is given for $N=11, C=0.16 / \mathrm{mm}$, $T=100 \mathrm{~mm}, x_{0}=-10$, and $\Delta=5.69$. As a consequence of the particular $z$ dependence of $C_{B}(z)$, the excitation becomes trapped at the last site for the remaining propagation. This is a very beneficial feature compared with transfer schemes based on $z$-independent Hamiltonians where PST can only be accomplished for one particular moment. The dynamic scheme thus removes the necessity of precise timing of the qubit extraction. One

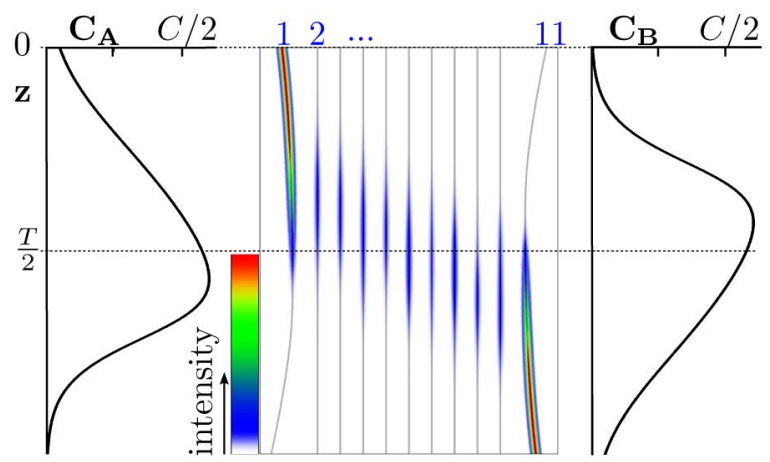

Fig. 2. Central image depicts the excitation dynamics in a waveguide structure with $N=11$ and $T=100 \mathrm{~mm}$. The plots to the left and right show couplings $C_{A}$ and $C_{B}$, respectively, as functions of the propagation direction. 
notices that the setting in Fig. 2 is reminiscent of a structure investigated recently in the context of stimulated Raman adiabatic passage (STIRAP) [16,17]. However, the transfer process itself is fundamentally different. It is characteristic for any STIRAP process for $C_{A}$ to reach its maximal value after $C_{B}$. Although in the example in Fig. 2 this is coincidentally fulfilled due to the short length of the chain being simulated, in general, the PST scheme does not require such a counter-intuitive sequence of the maxima of $C_{A}$ and $C_{B}[10]$. Furthermore, in the STIRAP process the light is transported via a "dark state," which is only existing if $N$ is an odd number. The PST scheme, however, is universal, meaning it works for all $N$. The excitation is traversing the homogeneous part and sequentially occupying all sites.

For our experiments, we fabricated various waveguide arrays in bulk fused silica glass chips by employing the femtosecond laser direct write technique [18]. All structures extend approximately $200 \mu \mathrm{m}$ in the lateral direction $x$ and they are fabricated to transfer the excitation to the receiver waveguide within $T=10 \mathrm{~cm}$. In our experiments, we inject horizontally (along $x$ ) polarized light with wavelength $\lambda=633 \mathrm{~nm}$ into the structure. The separation between the waveguides in the homogeneous part is $d_{h}=14.6 \mu \mathrm{m}$, which corresponds to $C=0.160 \mathrm{~mm}^{-1}$. In order to observe the evolution of the intensity distribution along the $z$ axis, we use fluorescence microscopy [19]. Figure 3(a) shows a transfer structure with 11 sites. The fraction of the total power measured in the receiver waveguide after transfer distance $T$ is taken as the transfer fidelity $(\mathcal{F})$. We achieve a fidelity of $\mathcal{F}^{\exp }=0.93$, whereas the theoretical value is $\mathcal{F}^{\text {theo }}=0.99$. The residual imperfection can be attributed to small deviations from the ideal coupling distribution resulting from an inaccuracy of the positioning system. Moreover, there is an inhomogeneity of the refractive index modulation along each waveguide and across the array especially at the edges of the array where the waveguide is influenced by the stress field of the neighbors only one-sidedly.

As our waveguides exhibit an elliptic shape, the fundamental mode fields depend on the polarization of the light. Consequently, the intersite coupling between the waveguides exhibits polarization dependence for a given spacing. In Fig. 4, couplings as a function of intersite spacing is shown, when the polarization is either vertical $\left(C_{V}\right)$ or horizontal $\left(C_{H}\right)$. This dependence has a strong

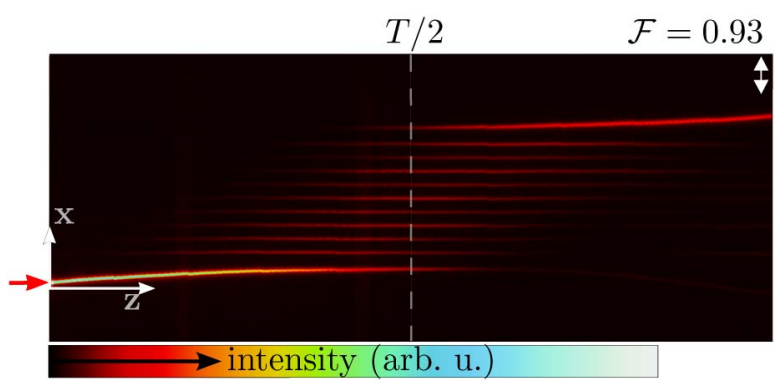

Fig. 3. Fluorescence microscopy images of the excitation transfer. The small red arrow shows the position of the light input. The horizontal polarization of the input light is indicated by the white arrow in the upper right corner. impact on time-independent transfer schemes, which are very sensitive to deviations of the couplings.

In order to exploit a new degree of freedom, we want to show how one can also operate on the polarization of the transferred light. By installing a supplementary waveguide close to the sender and receiver sites, henceforth called "ghost" waveguide, the polarization can be rotated. Note that the ghost waveguides exhibit a much higher refractive index than the other waveguides in the device, so that only their stress fields interact with the edge waveguides, but light is not able to couple into the ghosts [6]. It is shown in [20] that the laser writing induces a stress field that surrounds the ghost and causes a birefringent change of the refractive index of the neighboring waveguides. The strength of this induced birefringence is a function of the spacing between the ghost and the sender and receiver waveguides [20]. We additionally observe a rotation of the polarization of the guided light, which also depends on this separation. Therefore, the separation between the sender and receiver waveguides and their ghosts can be used to precisely tune the amount of polarization rotation. This offers, e.g., a possibility of fabricating integrated quantum gates.

For the experimental demonstration of polarization rotation during the PST, we fabricated transfer structures identical to the one analyzed above using the two additional ghosts. Importantly, the ghosts follow the same trajectory as sender and receiver guides. We launched horizontally polarized light into the sender waveguide and measured the (rotated) polarization as well as the transfer fidelity after postselecting that polarization. Our experimental results are summarized in Fig. $\underline{5}$. We were able to rotate the polarization of the light to up to almost $40^{\circ}$ by changing the spacing between the ghosts and the sender and receiver guides from $d_{\max }=18.6 \mu \mathrm{m}$ to $d_{\min }=12.6 \mu \mathrm{m}$ (red data points and black dashed line in Fig. 5). The inset in Fig. 5 shows the measured data for the normalized power in the receiver waveguide as a function of the rotation angle of the analyzer. The measurement corresponds to a separation of $14.6 \mu \mathrm{m}$ between the ghosts and edge. At $V+25^{\circ}$, the power is only $6 \%$ of the maximum value, which proves that the polarization

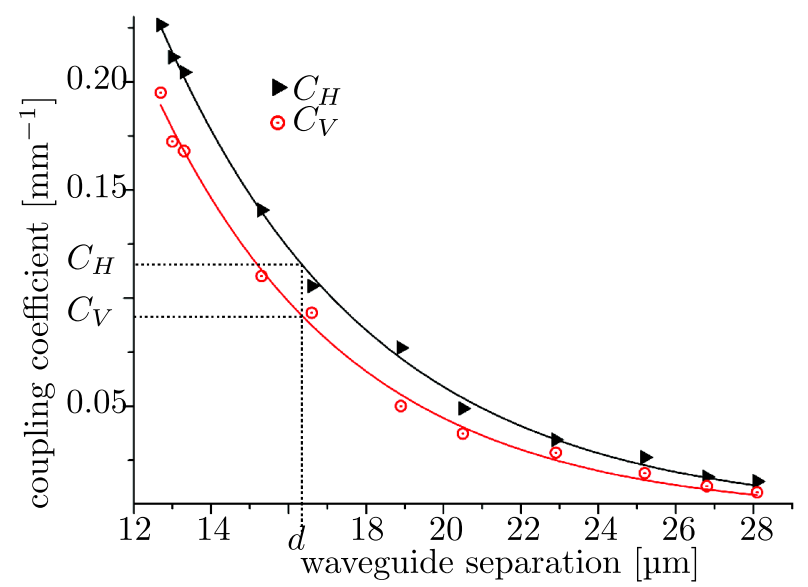

Fig. 4. Couplings $C_{V}$ and $C_{H}$ of vertically and horizontally polarized light, respectively, as functions of waveguide separation. The symbols are measured data, whereas the solid lines show the exponential fit according to Eq. (2). 


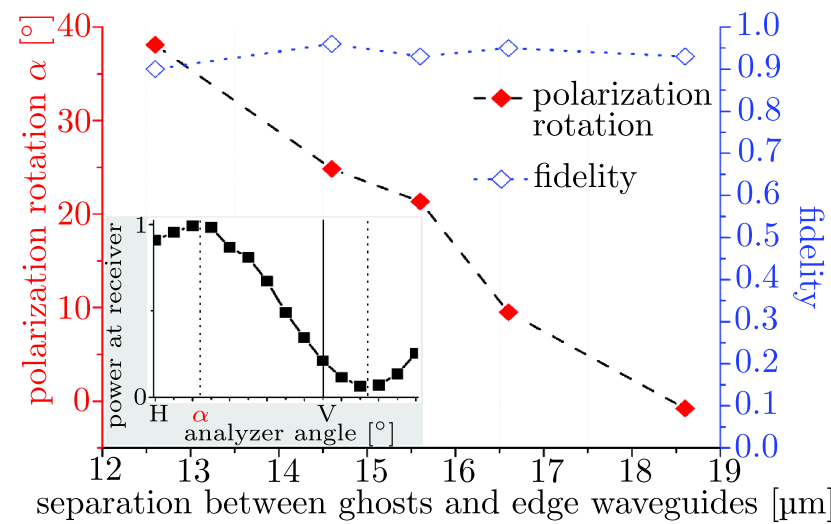

Fig. 5. Red data points show the relative angle of the analyzer $(\alpha)$ for which the power measured in the receiver waveguide is highest, i.e., the angle between the input and the output polarization, in dependence on the separation between the edge waveguides and the ghosts. Each of the blue data points gives the fidelity for that analyzer angle. The inset shows the power in the receiver waveguide as a function of the polarizer angle measured in the structure, with a $14.6 \mu \mathrm{m}$ separation between the ghosts and edge.

of the light in the receiver waveguide is again linear. No detectable polarization rotation occurred in absence of the ghosts. The transfer fidelity stays approximately constant and is always $>0.9$ (blue data points and blue dotted line in Fig. 5), despite the large polarization rotation. Note that the curvature of the sender and receiver waveguides was actually designed for one specific coupling $C$ in the homogeneous part and, therefore, only one polarization can be transferred with optimal fidelity. Hence, the transfer scheme is sufficiently robust to allow considerable deviations of the coupling without great degradation of transfer fidelity.

In conclusion, in our work we demonstrate the first implementation of a time-dependent PST scheme, using optical waveguides. The transfer structures exhibit great robustness to imperfections of the intersite coupling and overcome the need for precise extraction timing. Using the benefits of an optical setting, this allows the combination of photonic state transfer with well-controlled polarization rotation, which makes our device an adequate tool for coherent information transport and processing in quantum circuits as well as for applications in classical integrated optics.

This work was supported by the German Ministry of Education and Research (Center for Innovation Competence programme, grant no. 03Z1HN31) and the Thuringian Ministry for Education, Science and Culture (Research group Spacetime, grant no. 11027-514).

\section{References}

1. A. Steane, Rep. Prog. Phys. 61, 117 (1998).

2. T. D. Ladd, F. Lelezko, R. Laflamme, Y. Nakamura, C. Monroe, and J. L. O'Brien, Nature 464, 45 (2010).

3. S. Bose, Phys. Rev. Lett. 91, 207901 (2003).

4. M. Christandl, N. Datta, A. Ekert, and A. J. Landahl, Phys. Rev. Lett. 92, 187902 (2004).

5. M. Bellec, G. M. Nikolopoulos, and S. Tzortzakis, Opt. Lett. 37, 4504 (2012)

6. A. Perez-Leija, R. Keil, A. Kay, H. Moya-Cessa, S. Nolte, L.-C. Kwek, B. M. Rodriguez-Lara, A. Szameit, and D. N. Christodoulides, Phys. Rev. A 87, 012309 (2013).

7. A. Kay, Int. J. Quantum. Inform. 8, 641 (2010).

8. R. Ronke, T. P. Spiller, and I. D'Amico, Phys. Rev. A 83, 012325 (2011).

9. H. L. Haselgrove, Phys. Rev. A 72, 062326 (2005).

10. A. Kay, Phys. Rev. A 79, 042330 (2009).

11. A. Kay and P. J. Pemberton-Ross, Phys. Rev. A 81, 010301 (R) (2010).

12. S. Lloyd, A. J. Landahl, and J. J. E. Slotine, Phys. Rev. A 69, 012305 (2004).

13. A. Szameit, F. Dreisow, T. Pertsch, S. Nolte, and A. Tuennermann, Opt. Express 15, 1579 (2007).

14. T. J. Osborne and N. Linden, Phys. Rev. A 69, 052315 (2004).

15. H. S. Eisenberg, Y. Silberberg, R. Morandotti, and J. S. Aitchison, Phys. Rev. Lett. 85, 1863 (2000).

16. G. Della Valle, M. Ornigotti, T. Toney Fernandez, P. Laporta, S. Longhi, A. Coppa, and V. Foglietti, Appl. Phys. Lett. 92, 011106 (2008).

17. C. Ciret, V. Coda, A. A. Rangelov, D. N. Neshev, and G. Montemezzani, Phys. Rev. A 87, 013806 (2013).

18. A. Szameit and S. Nolte, J. Phys. B 43, 163001 (2010).

19. A. Szameit, F. Dreisow, H. Hartung, S. Nolte, A. Tuennermann, and F. Lederer, Appl. Phys. Lett. 90, 241113 (2007).

20. L. A. Fernandes, J. R. Grenier, P. R. Herman, J. S. Aitchison, and P. V. S. Marques, Opt. Express 20, 24103 (2012). 